\title{
FERROMAGNETISM IN Cr-BASED DILUTED MAGNETIC SEMICONDUCTORS
}

\section{J. BIINOWSKI}

Institute of Theoretical Physics, Warsaw University, Hoża 69, 00-681 Warszawa, Poland

$$
\text { P. KACMAN }
$$

Institute of Physics, Polish Academy of Sciences

Al. Lotników 32/46, 02-668 Warszawa, Poland

\section{AND J.A. MAJEWSKI}

Walter Schottky Institut, Technische Universität München Am Coulombwall, 85748 Garching, Germany

\begin{abstract}
It has been recently established that in zinc chalcogenides with $\mathrm{Cr}^{2+}$ paramagnetic ions the $p-d$ interactions are ferromagnetic. We clarify the origins of this property and make predictions concerning other $\mathrm{Cr}$-based diluted magnetic semiconductors. The observation of the ferromagnetic $p-d$ interaction resuscitated hopes for ferromagnetic $d-d$ exchange interactions in diluted magnetic semiconductors. We have calculated the nearest-neighbor superexchange integrals in $\mathrm{Cr}$-based diluted magnetic semiconductors. No simple correlation between the signs of $p-d$ and $d-d$ interactions has been found. Still, there are extencled energy regions where the calculated superexchange is ferromagnetic and the actual parameters of DMS with $\mathrm{Cr}^{2+}$ seem to match these regions.
\end{abstract}

PACS numbers: 71.70.Gm, 75.50.Pp, 75.50.Dd

The recent discovery of the ferromagnetic $p-d$ exchange interactions in zinc chalcogenides with $\mathrm{Cr}^{2+}$ ions [1] increased the interest in the studies of $\mathrm{Cr}$-based diluted magnetic semiconductors (DMS). The main objective of the present study is the theoretical, qualitative and quarititative, analysis of the superexchange interactions between $\mathrm{Cr}^{2+}$ ions in $\mathrm{A}_{\mathrm{II}} \mathrm{B}_{\mathrm{VI}}$ compounds within the same model as used previously for the calculation of the kinetic $(p-d)$ exchange in these materials [2]. To fix the proper values of the model parameters, we calculate numerical values of the $p-d$ exchange constants in zinc chalcogenides and compare them with the existing experimental data [3].

The $p-d$ interaction in $\mathrm{Cr}$-based $\mathrm{A}_{\mathrm{II}} \mathrm{B}_{\mathrm{VI}}$ compounds was shown $[2,4]$ to depend strongly on the relative population of $\mathrm{Cr}^{2+}$ ions with different tetragonal 
Jahn-Teller distortions. When none of the possible equivalent directions of these distortions is privileged, the cubic symmetry is in average preserved and, according to Eq. (30) in Ref. [2], the mean field $\ddot{p}-d$ exchange Hamiltonian for $\Gamma_{8}$ bands is proportional to $(2 B-C)$.

The exchange constant $B$ (given by Eq. (19) in Ref. [2]) collects the contributions from virtual transitions involving singly occupied ionic orbitals, i.e., the charge transfers from the ion into the valence band and from the valence band onto the ionic $d$-shell. In the following, the corresponding transfer energies we denote by $e_{1}$ and $e_{2}$, respectively. In Ref. [2], using arguments of the stability of $\mathrm{Cr}^{2+}$ ions, we suggested that the constant $B$ is negative, i.e., leads to antiferromagnetic $p-d$ interactions. As noted in Ref. [1], we had overlooked a possibility that $\mathrm{Cr}^{2+}$ ion might be stable not because of the energy reasons, $\left(e_{1}>0, e_{2}>0\right)$, but due to the absence of unoccupied states in the valence band, thus allowing for $e_{1}<0$.

The constant $C, \mathrm{Eq}$. (21) in Ref. [2], describes the contribution from virtual transitions involving the empty $t_{2 g}$ orbital only - the charge transfers from the valence band to the ground $(S=5 / 2)$ and excited $(S=3 / 2)$ states of $\mathrm{Cr}^{1+}$ ion, with the corresponding transfer energies $e_{2}-\Delta$ and $e_{2}$.

The model charge transfer energies $e_{1}$ and $e_{2}-\Delta$ can be approximately identified with those deduced from experimental data on the position of donor and acceptor $\mathrm{Cr}$ levels relative to the bands of the host material [6]. For the spin-excitation energy $\Delta$, we use the average free-ion value of $3 \mathrm{eV}$ [7]. The hybridization matrix element $V$ can be related to the anion orbital content in the $\Gamma_{8}$ wave functions and Harrison's parameters $V_{p d \sigma}$ and $V_{p d \pi}$ [8]. We impose the relation $V_{p d \pi}=-0.45 V_{p d \sigma}$ and for $V_{p d \sigma}$ we use the universal Harrison's values. The bands are described in the framework of Vogl's $s p^{3} s^{*}$ tight-binding theory [9] with the spin-orbit interaction [10], and the band parameters taken from Ref. [11].

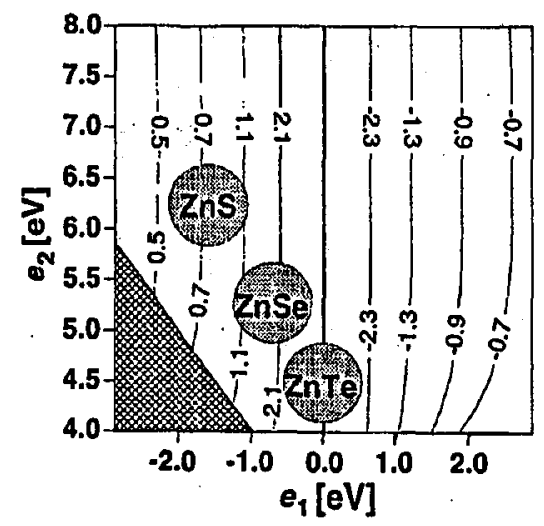

Fig. 1. Contour graph presenting. $\left(N_{0} \beta\right) /\left(A V_{p d \sigma}{ }^{2}\right)$ (in $\left.\left[\mathrm{eV}^{-1}\right]\right)$ as a function of transfer energies $e_{1}$ and $e_{2}$. The shaded circles define approximately the areas of the $e_{1}$ and $e_{2}$ values compatible with the positions of $\mathrm{Cr} 3+/ 2+$ and $2+/ 1+$ levels (for $\mathrm{ZnSe}, \mathrm{ZnS}$ and $\mathrm{ZnTe}$ ) given in Ref. [6]. The crosshatched triangle indicates the unphysical region of $e_{1}$ and $e_{2}$, where $e_{2}-\Delta+e_{1} \leq 0$. 
In all zinc chalcogenides the anion content $A$ and the parameter $V_{p d \sigma}$ enter $N_{0} \beta$ via multiplicative factors only. Thus, for comparison between various compounds we use an universal contour plot of $\left(N_{0} \beta\right) /\left(A V_{p d \sigma}{ }^{2}\right)$ vs. $e_{1}$ and $e_{2}$, as presented in Fig. 1. One can see that the theory correctly accounts for the observed ferromagnetic character of the $p-d$ exchange in $\mathrm{ZnSe}$ and $\mathrm{ZnS}$. Surprisingly enough, when the universal Harrison's values of $V_{p d \sigma}$ are used, the resulting $N_{0} \beta$ (see Table) for these compounds are in excellent agreement with expcrimental results [3]. ZnTe is situated at the borderline $e_{1}=0$ between very large ferromagnetic and very large antiferromagnetic exchange constants. This scems to be in qualitative agreement with the unusually large ferromagnetic exchange constant observed recently in $\mathrm{Cr}$ doped $\mathrm{ZnTe}[5]$. Moreover, our result indicates that one can expect unusual concentration and pressure dependences of the $p-d$ exchange constant in this material, even though the use of the perturbation might be questionable in the case $e_{1}=0$.

To calculate the superexchange, one has to determine the fourtli-order perturbation matrix for the hybridization Harniltonian applied to the system of two ions in a crystal with completely filled valence bands. The effective spin-spin interaction between two $\mathrm{Cr}^{2+}$ impurity ions occupying two crystal sites separated by the vector $\boldsymbol{R}_{12}$ we describe by the following operator:

$$
\widehat{H}_{S}=-2 \sum_{\alpha, \beta} J_{\alpha \beta}^{\gamma \delta}\left(\boldsymbol{R}_{12}\right) \widehat{S}_{1 \alpha} \widehat{S}_{2 \beta},
$$

where $\widehat{S}_{i \alpha}$ is the $\alpha$ th component $(\alpha=x, y, z)$ of the spin operator of the ion $i$. The indices $\gamma$ and $\delta$ define the Jaln-'Teller singlet states of the interacting ions. We obtain the superexchange tensor " $J$ consisting of three terms coming from: occupied orbitals of the two interacting ions $(F)$; occupied orbitals of one ion and the empty orbital of the second ion $(H)$; and empty orbitals of both ions $(G)$. The contributions to the superexchange tensor coming from the occupied orbitals only $(F)$ and those involving the empty orbitals $(H$ and $G$ ) differ in sign.

We have performed numerical calculations of the superexchange tensor for the $\mathrm{Cr}^{2+}$ ions in $\mathrm{ZnS}, \mathrm{ZnSe}$ and $\mathrm{ZnTe}$ crystals (the Harrison's parameter $V_{s d \sigma}=$ $1.08 V_{p d \sigma}$ had to be also taken into account). The values of the diagonal tensor component $J_{x x}^{\gamma \delta}$ (with $(\gamma, \delta)=(x y, x y),(x y, y z),(y z, y z)$, and $\left.(y z, x z)\right)$ for the two nearest-neighbor $\mathrm{Cr}^{2+}$ ions lying in the $z=0$ plane are presented in Table. The off-diagonal tensor components and the differences in the diagonal matrix elements, proportional to the spin-orbit splitting of the valence band, are smaller

TABLE

The dominant superexchange tensor components in zinc chalcogenides (in [K]).

\begin{tabular}{c|c|c|c|c|c|c|c}
\hline \hline & $e_{1}[\mathrm{eV}]$ & $e_{2}[\mathrm{eV}]$ & $N_{0} \beta$ & $J_{x x}^{x y, x y}$ & $J_{x x}^{y z, y z}$ & $J_{x x}^{x y, y z}$ & $J_{x x}^{x z, y z}$ \\
\hline $\mathrm{ZnS}$ & -1.7 & 6.2 & $0.48,0.57 \pm 0.1 \mathrm{e}$ & 0.55 & 1.06 & 0.06 & 0.37 \\
$\mathrm{ZnSe}$ & -0.75 & 5.25 & $0.91,0.83 \pm 0.1 \mathrm{e}$ & 0.62 & 1.20 & 0.40 & 0.71 \\
$\mathrm{ZnTe}$ & 0.0 & 4.5 & $\pm \infty$ & 0.33 & 0.80 & 0.42 & 0.67
\end{tabular}


than the diagonal components by one and two orders of magnitude, respectively. All diagonal components in all zinc chalcogenides turned out to be positive, i.e., the positive contributions from empty orbitals prevail over the Mn-like negative contribution from the occupied orbitals. $\Lambda$ ccording to the definition (1), positive exchange constants correspond to the ferromagnetic interactions.

In summary, we have tested the model by explaining the observed ferromagnetic $p$ - $d$ exchange constants in $\mathrm{Cr}$-based zinc chalcogenides. The superexchange between two nearest-neighbor $\mathrm{Cr}^{2+}$ ions obtained within the same model is dominated by the isotropic Heisenberg-type interaction, ferromagnetic in all materials studied. The strength of the interaction depends on the relative orientations of the Jahn-Teller distortions of the interacting chromium ions, so that the $d-d$ interactions in Cr-based DMS may not be characterized by the single nearest-neighbor exchange constant $J_{1}$. Similar properties of both $p-d$ and $d-d$ exchange are expected in cadmium chalcogenides, for which the transfer energies are almost identical as in the corresponding zinc compounds.

This work was partially supported by the grant 2 P302 17907 of the State Committee for Scientific Research (Republic of Poland).

\section{References}

[1] W. Mac, Nguyen The Khoi, A. Twardowski, J.A. Gaj, M. Demianiuk, Phys. Rev. Lett. 71, 2327 (1993).

[2] J. Blinowski, P. Kacman, Phys. Rev. B 46, 12298 (1992).

[3] W. Mac, Nguyen The Khoi, A. Twardowski, in: Proc. 22nd Internat. Conf. on the Physics of Semiconductors, Vancouver (Canada) 1994, Ed. D.J. Lockwood, World Scientific, Singapore 1995, p. 2573.

[4] J. Blinowski, P. Kacman, Mater. Sci. Forum 83-87, 523 (1992).

[5] W. Mac, private communication.

[6] J.M. Langer, C. Delerue, M. Lannoo, II. IIeinrich, Phys. Rev. B 38, 7723 (1988); J. Kossut, W. Dobrowolski, in: Handbook of Magnetic Materials, Ed. K.H.J. Buschow, Vol. 7, North-Holland, Amsterdam 1993, p. 231; T. Dietl, in: Handbook on Semiconductors, Ed. T.S. Moss, Vol. 3b, North-Holland, Amsterdarn 1994, p. 1251.

[7] Ch.E. Moore, Atomic Energy Levels, Vol: II, National Bureau of Standards, Circular 467, 1952.

[8] W.A. Harrison, Electronic Structurc and the Properties of Solids, W.H. Freeman and Co., San Francisco 1980.

[9] P. Vogl, H.P. Hjalmarson, J.D. Dow, J. Chem. Solids 44, 365 (1983).

[10] A. Kobayashi, O.F. Sankey, J.D. Dow, Phys. Rev. B 25, 6367 (1982).

[11] D. Bertho, D. Boiron, A. Simon, C. Jouanin, C. Priester, Phys. Rev. B 44, 6118 (1991-II). 